\title{
The Implication of the Corticotropin Releasing Factor in Nicotine Dependence and Significance for Pharmacotherapy in Smoking Cessation
}

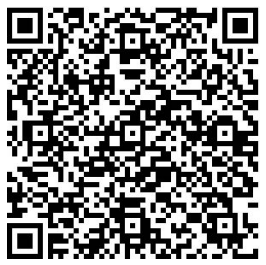

\author{
Vasileia Karasavva ${ }^{1 *}$
}

Smoking represents one of the greatest preventable causes of death globally, and pharmacological treatments of higher efficacy targeting smoking cessation are necessary. Current drug interventions show only modest success rates and do not adequately address nicotine withdrawal-induced anxiety that is heavily implicated in relapse and failed quit attempts. The purpose of this paper is to highlight that nicotine dependence is at least partially maintained through the negative reinforcing effect of avoiding abstinence-induced anxiety. This paper presents findings which suggest that this effect is mediated by the activation of the Corticotropin Releasing Factor (CRF) system are presented and the implications of a therapeutic agent containing a $\mathrm{CRF}_{1}$ antagonistare discussed. Specifically, $\mathrm{CRF}_{1}$ blockers are highlighted as alternatives for individuals with multiple failed quit attempts because they target the abstinence-induced increased anxiety that seems to lie at the core of failed cessation attempts.

\section{INTRODUCTION}

Addiction is often conceptualized as the loss of control over reward-seeking behaviors that can ultimately lead to the emergence of a cluster of cognitive, psychological and behavioral symptoms (American Psychiatric Association, 2013). It is marked by the need to seek and consume the drug of choice, an inability to limit the intake and the emergence of a distinct withdrawal syndrome in abstinence (Wise and Koob, 2014). The hallmark of addiction is the motivational switch from expectancy and euphoria when using, to the anxiety, dysphoria and compulsion that accompany even brief abstinence (Wise and Koob, 2014). In other words, initially, drug use is maintained through positive reinforcement by the rewarding properties of the drug of abuse. However, as dependency increases, it is maintained through negative reinforcement by eliminating, and ultimately, completely avoiding withdrawal symptoms (Wise and Koob, 2014). This motivational switch could be a potential target of new pharmacological interventions in substance use disorder (SUD).

Emotional responses, such as drug use, elicit two processes: the primary a-process that mediates the rewarding properties of the stimulus but shows signs of habituation and the opponent b-process, which has the opposite effect of the a-process and counteracts

${ }^{1}$ Department of Neuroscience, Carleton University, Ottawa, Ontario, Canada K1S 5B6

*To whom correspondence should be addressed: vasiakaras@gmail.com

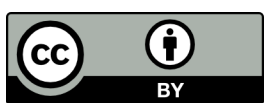

Except where otherwise noted, this work is licensed under https://creativecommons.org/licenses/by/4.0

doi:10.22186/jyi.36.3.36-43 it (Skinner and Aubin, 2010). Drug use initially causes an acute release of dopamine from the nucleus accumbens (NAc) and initiates the release of opioids, producing a rewarding affective state (Koob and Volkow, 2010). However, this drug-induced reward is opposed by processes that reduce drug effects and attempt the return of hedonic states to homeostasis (Koob and Volkow, 2009). This is done through the activation of the brain's stress system, the corticotropin releasing factor (CRF) system that then signals to the extended amygdala and hippocampus to elicit a stress response (Koob and Volkow, 2009). Thus, repeated use and activation of the opponent b-processes leads to a reduction in drug effects resulting in the development of tolerance and withdrawal symptoms in abstinence (Skinner and Aubin, 2010). Taken together, the compulsive nature of addiction can thus be viewed as a cycle of increasing dysregulation of brain reward and anti-reward mechanisms that results in a shifting hedonic baseline due to b-processes failing to return to normal homeostatic range (Garavan et al., 2000). In other words, chronic drug use leads to the elevation of reward thresholds that do not return to baseline during abstinence, leading to marked dysphoria and anhedonia, i.e. negative affect and an inability to experience pleasure (Garavan et al., 2000). Current research has shifted its focus to investigate how avoiding those dysphoric feelings produced in abstinence acts as a powerful motivator for continuous use through negative reinforcement. Thus, a paradox emerges in developing effective drug rehabilitation treatments as abstinence is both the ultimate goal and one of the reasons behind compulsive use.

The development of an effective treatment is particularly important for smoking, since cigarette smoking constitutes a major health risk factor and is the leading cause of preventable deaths 
in the Western world (Baliunas et al., 2007). In Canada, 16.6\% of deaths can be attributed to smoking-related illness, such as malignant neoplasms, cardiovascular disease (CVD), respiratory disease, lung cancer and chronic obstructive pulmonary disease (COPD) (Baliunas et al., 2007). Although smoking rates are at a decline, this is mainly because of a lower incidence of initiating smoking and lower smoking rates among younger people (US Department of Health and Human Services, 2014). In 2001, the rate of smoking among Canadian youth 15 years and older was 25\%, whereas in 2012, this number had dropped to $16 \%$ (Health Canada, 2017). However, the incidence of attempts to quit smoking has remained relatively stable, with only $2 \%$ of quit attempts becoming successful each year, despite the fact that $60 \%$ of surveyed smokers claimed they wished to quit smoking within the next six months (Malarcher et al., 2011; Ontario Tobacco Research Unit, 2013). In fact, most attempts to quit smoking only last on average seven days, and most smokers go through the transitions of smoking, reduction in smoking, and abstinence multiple times, with some research suggesting that the number of times it takes to quit successfully is as high as 30 (Chaiton et al., 2016; Hughes et al. 2014).

The addictive properties of smoking are mainly due to nicotine: a colourless, volatile alkaloid found in cigarettes (Dietz, 2016). A typical cigarette contains $6 \mathrm{mg}$ to $11 \mathrm{mg}$ of nicotine, though only about a third of that actually reaches the smoker's bloodstream depending on the number and length of puffs inhaled (Feldman et al., 1997). Nicotine readily passes through the absorbent surface of the lungs to the bloodstream and then crosses the blood brain barrier (BBB) rapidly stimulating the brain's reward pathways and thus providing instant reward (Benowitz, 2008). This is due to the activation of the dopaminergic mesolimbic tract and specifically the induction of burst firing in ventral tegmental area (VTA) neurons, resulting in enhanced dopamine release in the NAc (Feldman et al., 1997). Smoking behaviors are often triggered by environmental cues through classical conditioning, comparable to alcohol consumption and eating, which also contribute to their addictive nature (Caggiula et al., 2008). Additionally, it has to be noted that the highly addictive nature of smoking is at least partially due to the high number of reinforcements a single cigarette yields (Feldman et al., 1997). This is because each puff acts a separate "hit" of nicotine, which reaches the brain within seconds providing a rewarding burst of dopamine in the NAc (Feldman et al., 1997). Finally, nicotine is metabolized and eliminated quickly and thus, has a rapid onset of withdrawal that is characterized by an anxious and dysphoric affective state (Benowitz, 2008). For people dependent on nicotine, even a brief abstinence of a few hours triggers feelings of craving accompanied by a growing urge to smoke, difficulty concentrating, irritability, and restlessness (Breslau et al. 1992). This rapid onset of withdrawal is suggested to motivate smokers to eliminate its negative symptoms and end the abstinence period (Feldman et al., 1997). Therefore, the CRF system, which is thought to be responsible for the abstinence-induced dysphoria in substance use (Cohen et al., 2013), could potentially be implicated in nicotine withdrawal and dependence, and ultimately provide a target for pharmacological intervention in nicotine cessation.

The purpose of this review is to demonstrate that nicotine addiction is at least partially maintained via abstinence-induced anxiety that activates the CRF system and can thus be mediated by pharmacological interventions that target and block CRF receptors. More specifically, the purpose of this paper is to (a) review current pharmacological approaches in smoking cessation, (b) examine the relationship between nicotine withdrawal and activation of the CRF system, and (c) explore the potential of CRF blockers for smoking cessation.

\section{CURRENT PHARMACOLOGICAL APPROACHES IN SMOKING CESSATION}

Usually the first line of treatment in nicotine cessation is nicotine replacement therapy (NRT), which aims to reduce the abstinenceinduced anxiogenic symptoms during the first eight to twelve weeks of quitting, when they are the most severe (Etter and Stapleton, 2006). NRT replaces cigarette-derived nicotine with other pharmacological alternatives, often in the form of nicotine patches or gum, and is particularly popular mainly because it is safe, economic and easily accessible as an over the counter medication (Etter and Stapleton, 2006). Both nicotine gum and nicotine patches have been shown to provide relief from craving and withdrawal (Christen et al., 1991; Fagerström et al., 1993). However, the lifetime and cost-efficacy of such treatments is often overestimated as the relapse rate between NRT and other treatments does not differ significantly (Etter and Stapleton, 2006). In addition, NRTs target and address smoking cessation, but do little to address the core problem of nicotine dependence (Schnoll et al., 2015).

Alternatively, bupropion, an atypical antidepressant, is also licensed for smoking cessation therapy and can be recommended as a first-line agent for smoking cessation (Hughes et al., 2014). When used for smoking cessation, bupropion reduces the severity of the nicotine withdrawal syndrome symptoms, including abstinence-induced depression, irritability, difficulty concentrating, and craving, while increasing the likelihood of successful cessation within the first three months of its prescription (Shiffman et al., 2000; Wilkes, 2008). Thus, bupropion is thought to be particularly effective in the early stages of smoking cessation and likely works by stimulating the dopaminergic system and allowing DA to remain in the synaptic cleft for a longer duration (Shiffman et al., 2000; Wilkes, 2008). However, it is unclear how long those effects remain after the cessation of bupropion treatment (Wilkes, 2008). In fact, bupropion treatment only shows modest long-term success rates one year after its prescription, with successful cessation rates averaging from $7 \%$ to $30 \%$ depending on the level of adjunctive behavioral counseling, leaving ample room for improvement (Nides et al., 2006). Finally, some health concerns have been raised over the use of bupropion as it has been associated with increased risk of seizures. As a result, its prescription rates have decreased over the last few years (Hubbard et al., 2005).

The limited success rates of currently available therapies led 
investigators to develop a therapeutic agent specifically for smoking cessation. Varenicline is a non-nicotine agent that selectively binds and agonizes the $\alpha 4 \beta 2$ nicotinic acetylcholine receptor (Nides et al., 2006). This receptor plays an important role in the rewarding properties of nicotine, which binds to it with high affinity, resulting in an increase in dopamine release in the NAc (Subramaniyan and Dani, 2015). Varenicline significantly reduces cravings for smoking as well as ratings of satisfaction after the first cigarette following a period of abstinence in humans (West et al., 2008). The efficacy of varenicline was systematically tested in two large, randomized, double-blind Phase III clinical trials that used similar methodologies (Nides et al., 2006; Gonzales et al., 2006). In both studies, participants were administered varenicline, bupropion or placebo treatment for 12 weeks and were then followed up 40 weeks later to assess the success rate of each treatment (Nides et al., 2006; Gonzales et al., 2006). The results showed that compared to bupropion or placebo varenicline treatment had higher efficacy and increased prolonged abstinence rates (Nides et al., 2006; Gonzales et al., 2006). However, serious concerns have been raised over the adverse side effects of varenicline. Specifically, the US Food and Drug Administration (FDA) has published reports of significant neuropsychiatric adverse events including suicidal ideation, depression and mood disturbances following treatment with varenicline (US FDA, 2010). An association between varenicline and risk of cardiovascular events, both ischemic and arrhythmic, raises additional safety concerns (Singh et al., 2011). The Committee to Evaluate Drugs (CED) supports the prescription of varenicline in Canada and recommended its public funding as a Limited Use Benefit on the Ontario Drug Benefit Formulary while simultaneously noting that more research is needed to investigate the association between varenicline and myocardial infractions and cerebrovascular accidents (Committee to Evaluate Drugs, 2012).

The idea of nicotine vaccinations has gained more interest over the last few years. One way nicotine vaccinations can work is through active immunization, which requires repeated administration of the immunogen, in this case nicotine, to the individual so the appropriate antibodies can be produced (Cornish et al., 2011). Alternatively, in passive immunization, the immunogen is administered in a different species that produces antibodies against it (Cornish et al., 2013). Those antibodies are transferred at a later date to the individual via vaccination (Cornish et al., 2013). However, because nicotine itself is not immunogenic and thus cannot produce a strong enough immune response, smoking is not sufficient for the production of nicotine antibodies (Pentel and Lesage, 2014). Thus, the use of recombinant DNA technology that binds nicotine to different carrier conjugate proteins is necessary (Pentel and Lesage, 2014). Each conjugate protein can function as an independent immunogen, and so using multiple conjugate proteins concurrently can trigger high antibody concentrations (Pentel and Lesage, 2014). The antibodies can then bind on the conjugate protein-nicotine complex and prevent and/or slow down its crossing of the blood brain barrier and entering the brain, thus reducing the reinforcing effects of nicotine (Pentel and Lesage, 2014). Nicotine vaccination trials have produced some promising results in animals thus far and have been shown to reduce nicotine distribution to the brain as well as decreasing the rate by which nicotine crosses the blood brain barrier, making it presumably less reinforcing (Pentel et al., 2006; Pravetoni et al., 2011). One clinical trial found that the use of nicotine immunization in human, current smokers is safe and moderately effective (Hatsukami et al., 2005). Some of the methodologies used in such trials have been questioned over their small sample size and extensive use of animal models. In addition, concerns over their previous reproducible positive results have been raised as clinical trials in humans. They have not been able to replicate the results or even the preclinical findings. In fact, all Phase III trials reported to date suggest that nicotine immunization is ineffective (Giri et al., 2017). The most applicable example is NicVax, an experimental conjugate nicotine vaccine with initially encouraging early trials that, however, showed efficacy no better than that of a placebo in two successive phase III trials (ZalewskaKaszubska, 2015).

This section described some of the most popular pharmacological interventions that are used in smoking cessation. Current therapeutic treatments only show modest results while some of them are also associated with significant health concerns. This includes both bupropion as well as varenicline which have been associated with the incidence of serious side-effects and have shown modest efficacy, questioning their cost-reward ratio. Nicotine vaccines provide a safer alternative, but because nicotine does not produce a strong enough immune response, recombinant DNA technology is required. With such a costly production process and limited successful results, nicotine immunization appears to be a disappointing direction. On the other hand, replacement therapies are a safer and more economical alternative but do not directly address nicotine dependence since their goal is smoking cessation rather than treatment of nicotine addiction. In addition, they have limited success rate of $20 \%$ even after long-term NRT (Schnoll et al., 2017). Taken together, a comprehensive treatment that has a direct effect on the anxiogenic, negative affect brought on by nicotine withdrawal is necessary to not only facilitate smoking cessation but also to treat the problem at its core and reduce nicotine dependency.

\section{THE SWITCH FROM IMPULSIVE TO COMPULSIVE USE}

As mentioned, initial drug use initiates a-processes that facilitate reward and intoxication, but as time progresses, b-processes that are longer lasting and have the opposite effect take over (Skinner and Aubin, 2010). Chronic activation of b-processes leads to system neuroadaptations (Koob and Volkow, 2010).

Specifically, within system adaptations refer to changes in the same brain areas that also mediate the rewarding properties of drugs of abuse (Koob and Volkow, 2010). Such neuroadaptations include attenuated activity of the mesolimbic dopaminergic system and reduced dopaminergic and serotonergic signaling of the NAc during withdrawal (Koob and Volkow, 2010). Those changes are consistent with the symptomatology exhibited during 
withdrawal, including feelings of dysphoria, irritability, and loss of interest for natural rewards (Koob and Volkow, 2016). In addition, individuals with SUD show a significant reduction in D2 receptors in the striatum that often persists even months after detoxification (Johnson and Kenny, 2010; Volkow et al., 2009). This reduction in D2 receptors has a marked effect on the prefrontal cortex glucose metabolism and is associated with increased impulsivity and craving (Koob and Volkow, 2016). Thus, within system adaptations play an important role in the abstinence-induced emotional dysregulation that is experienced after chronic use.

On the other hand, between-system neuroadaptations refer to changes in brain areas that are not directly involved with the rewarding properties of drugs of abuse and are usually associated with stress regulation (Koob and Volkow, 2010). Drug use and its subsequent dopaminergic activation of the mesolimbic pathway also increases the levels of dynorphin, a class of opioids that is strongly associated with the dysphoric elements of stress, in the NAc (Koob, 2008). Binding of dynorphin on the endogenous $\kappa$-opioid receptor inhibits dopaminergic activity and is associated with the aversive symptoms of withdrawal in multiple drugs of abuse, including cocaine, ethanol, and opioids (Koob, 2008). Another between-system activation is the protracted activation of the extended amygdala by CRF (Macenski, 2012). In fact, CRF activation in the amygdala is observed in all drugs of abuse, suggesting CRF activation is an integral part of the motivational switch to compulsive use (Koob and Volkow, 2010). Another common between-system neuroadaptation is the over-activation of the hypothalamic-pituitary-adrenal (HPA) axis during withdrawal which is observed in most major drugs of abuse (Silva and Madeira, 2012). More specifically, both acute and prolonged exposure to drugs activates the HPA axis (Lukoyanov et al., 1999). This happens by increasing the levels of adrenocorticotrophic hormone (ACTH) and corticosterone (Madeira and Paula-Barbosa, 1999). The end result is the synthesis of CRF suggesting the adaptation of the HPA to substance use (Madeira and Paula-Barbosa, 1999). Those changes seem to be long-lasting and still present during protracted withdrawal in animal models of cocaine and ethanol dependence that showed elevated levels of CRF six weeks after the last self-administration (Zorrilla et al., 2001). Additionally, some research suggests that along with the increased activation of the CRF, decreased activation of Neuropeptide Y (NPY) is also observed during withdrawal. Administration of NPY into the amygdala is thought to have anxiolytic effects as it has been shown to be associated with a reduction in drug intake in ethanol dependent rats (Slawecki et al., 2005; Thorsell et al., 2007).

Taken together, these results support the motivational significance of the dysregulation of the between-system neuroadaptations in SUD. In particular, increased activation of the brain stress system (CRF, dynorphin) along with reduced activity of anxiolytic brain agents like the NPY seem to be of particular importance in the motivational switch observed in dependence. Therefore, a pharmacological intervention reversing those effects by either reducing the effect of anxiogenic agents or increasing the effect of the anxiolytic ones could facilitate protracted abstinence.

\section{CRF AND NICOTINE DEPENDENCE}

CRF is a neuropeptide that is expressed both in the periphery, including the lungs, blood vessels, the gonads and across the central nervous system (Vale et al., 1981). It is established as one of the major moderators of the HPA axis activity by stimulating the production and secretion of Adreno Corticotropin Releasing Factor (ACTH) from the pituitary gland (Vale et al., 1981). There are two identified CRF receptors: the $\mathrm{CRF}_{1}$ and $\mathrm{CRF}_{2}$ receptor. Of those two receptors, $\mathrm{CRF}_{2}$ is thought to play a more brain region-dependent role with some research findings suggesting it is involved in inhibiting adrenocortical functioning on the Central Nervous System (CNS; Henry et al., 2006; Zhao et al., 2007). In contrast, over-activation of the $\mathrm{CRF}_{1}$ receptor in the limbic system is linked with depression and anxiety, potentially explaining why $\mathrm{CRF}_{1}$ antagonists have yielded antidepressant effects (Künzel et al., 2003). Thus, the two receptors seem to have opposite effects, with binding on $\mathrm{CRF}_{1}$ having an anxiogenic effect whereas binding on $\mathrm{CRF}_{2}$ has an anxiolytic effect. In the peripheral nervous system, CRF influences the synthesis and release of both cortisol and catecholamines, thus playing a big role in the activation of the sympathetic nervous system (Tsatsanis et al., 2007). In the brain, the areas with the highest expression of CRF include the paraventricular nucleus of the hypothalamus (PVN), which secretes CRF during the activation of the HPA axis stress response and the extended amygdala that is implicated in negative affect and fear response (Boorse and Denver, 2006, Smith and Vale, 2006,). Those CRF projections to extrahypothalamic structures eventually reach and influence the activity of the VTA and NAc in the mesolimbic reward pathway (Rodaros et al., 2007). Thus, it is well established by research that the CRF system plays an important role in anxiety and negative affect as well as in inhibiting the brain reward system (Bruijnzeel et al., 2012; Grieder et al., 2014).

In addition, research suggests that over-activation of the CRF system is also implicated in SUDs, particularly during the withdrawal stage (Mason et al., 2012). Animal studies have shown that acute alcohol withdrawal is accompanied by a marked increase in CRF concentration in the striatum (Olive et al., 2002). Similar increases in CRF were also found during cocaine, heroin and cannabinoid withdrawal, which presumably hints at a similar increase in CRF during nicotine withdrawal across all SUDs (Mason et al., 2012; Park et al., 2013; Richter et al., 1999). Finally, recent studies have identified the significance of the CRF system in nicotine dependence and stress-induced relapse (Cohen et al., 2015). Specifically, there is evidence strongly supporting the importance of the CRF system and stress during nicotine acquisition, maintenance, and relapse (Kutlu et al., 2016). High levels of stress during adolescence, the most important time period for the development of chronic smoking habits, is associated with higher rates of smoking initiation (Kutlu et al., 2016). Childhood abuse (Nichols and Harlow, 2004), adverse childhood experiences including physical and psychological neglect of basic need (Ramiro et al., 2010), ethnic 
discrimination (Brondolo et al., 2015), poor academic performance (O'Loughlin et al., 2014), dropping out of school, low academic aspirations and low socioeconomic status (Pedersen and Soest, 2017) are all associated with higher incidence of smoking uptake during adolescence and were indicators of subsequent higher rates of smoking in adulthood. As established in the literature, stress activates the CRF system, usually through the $\mathrm{CRF}_{1}$ receptor. Research has also shown that such activation during adolescence enhances the rewarding properties of nicotine, exemplifying the importance that CRF system activation plays in initiating smoking behavior (Brielmaier et al., 2012; Bruijnzeel, 2012).

Stress and activation of the CRF system plays an equally important role in withdrawal and could be the main reason why $95 \%$ of the people who attempt to quit smoking fail and relapse (Garvey et al., 1992). Symptoms of nicotine withdrawal are psychological rather than physiological in nature and include increased irritability, restlessness, anxiety, difficulty concentrating, insomnia as well as feelings of anger and have a mean duration of two to four weeks (Hughes et al., 2007). Research has shown that experiencing stress and feelings of anxiety during abstinence decreases the ability to resist smoking and increases both the frequency of smoking as well as the perceived feelings of satisfaction after lapsing (Cohen et al., 2013; McKee, 2011). Therefore, a hard-to-break cycle of abstinence-induced anxiety and relapse is created with an overactive CRF system at its center. Nicotine abstinence induces an increase of the CRF concentration in the central nucleus of the amygdala (CeA), which is thought to be the moderator of the emotional symptoms of withdrawal (George et al., 2007). This overactivation of the CRF system is also associated with the induction of a negative emotional state that in turn induces craving and nicotine seeking behavior and increased self-administration of nicotine (Bruijinzeel et al., 2012; Cohen et al., 2013; George et al., 2007). Furthermore, it has also been demonstrated that nicotine dependence up-regulates the production of CRF in the Ventral Tegmental Area (VTA) and alters its rate of firing (Grieder et al., 2014). Using immunohistochemistry, researchers were able to show that nicotine abstinence is strongly associated with the depletion of CRF in the VTA (Grieder et al., 2014). They were also able to reverse abstinence-induced motivational effects including anxiety-like behavior and increased nicotine consumption by down-regulating the expression of CRF (Grieder et al., 2014). These results are promising as they present strong evidence supporting the involvement of the CRF system in the anxiety-like symptoms experienced during withdrawal and concurrently show that reversal of this effect is possible by down-regulating the activity of this system.

In addition, in a recent animal study, researchers were able to prevent abstinence-induced anxiety with the administration of MPZP, a $\mathrm{CRF}_{1}$ receptor antagonist, in the VTA showcasing the potential therapeutic value of $\mathrm{CRF}_{1}$ antagonists (Grieder et al., 2014). Other animal studies replicated those results and showed that the administration of MPZP in other brain areas, like the VTA or the NAc, that are also heavily implicated in nicotine withdrawal like $\mathrm{CeA}$ also reduces abstinence-induced anxiety and hypearlge- sia (Cohen et al., 2013). Specifically, access to nicotine was first shown to mediate higher breaking points in a progressive ration of reinforcement as well as increased levels of anxiety and mechanical hypersensitivity during abstinence in rats (Cohen et al., 2013). These effects, however, were reversed when MPZP was infused in the CeA (Cohen et al., 2013). The use of MPZP has also shown to be useful in attenuating the abstinence-induced increased rates of self-administration of nicotine and nicotine seeking behaviors (George et al., 2007). Similar results were obtained with the administration of a different $\mathrm{CRF}_{1}$ antagonist, mecamylamine, in the same brain area, the CeA, highlighting how the blockade of this receptor with any antagonist is a good way to diminish dysphoric feelings experienced during nicotine withdrawal (Bruijinzeel et al., 2012). The brain area those antagonists are administered in also plays a role as blockade of $\mathrm{CRF}_{1}$ receptors in the NAc and CeA appear to be effective, but blocking either $\mathrm{CRF}_{1}$ or $\mathrm{CRF}_{2}$ receptors in the bed nucleus of the stria terminalis (BNST) is not (Bruijnzeel, 2016). In general, blockade of the $\mathrm{CRF}_{2}$ receptor does not seem to produce similar therapeutic effects in reducing anhedonia and even increased anxiety-like behaviors (Kamdi et al., 2009; ManoOtagiri et al., 2009). Those animal studies suggest that inhibiting the CRF system with the use of selective $\mathrm{CRF}_{1}$ receptor antagonist poses a potential useful method of reducing anxiety experienced during withdrawal and consequently reducing nicotine seeking behaviors and the likelihood of relapse.

To the best of our knowledge, no $\mathrm{CRF}_{1}$ antagonists have been used in a preclinical or clinical trial for smoking cessation in humans. However, there is a growing interest surrounding the use of $\mathrm{CRF}_{1}$ antagonists for a multitude of stress-related disorders and results from such studies could be useful for the design of a therapeutic agent that inhibits the CRF system with the goal of reducing anxiety during withdrawal (Murrough et al., 2015). The blockade of $\mathrm{CRF}_{1}$ antagonists in the amygdala that has been investigated extensively in animal models and shown to be an effective way of reducing abstinence-induced anxiety and levels of self-administration in reinstatement has also been studied in humans. Specifically, the $\mathrm{CRF}_{1}$ receptor antagonist R31753 was shown to produce a dose-dependent decrease in the metabolism of glucose in the amygdala of healthy male participants, suggesting a decrease in its activity levels (Schmidt et al., 2009). In a randomized, double-blind, placebo-controlled study, inhalation of the same $\mathrm{CRF}_{1}$ antagonist (R31753) was associated with reduced levels of anxiety (Bailey et al., 2011). The promising nature of those results in addition to how $\mathrm{CRF}_{1}$ antagonists usually make them a good option for the treatment of nicotine addiction that targets the anxiety-like symptoms that are induced during withdrawal. That being said, more pre-clinical and clinical studies are warranted to investigate the use of such antagonists in humans who attempt to quit smoking.

\section{DISCUSSION}

Despite the decrease in the number of smokers in the past decade, smoking's burden of disease remains staggering and more efficient 
therapeutic interventions for smoking cessation are necessary. Past research strongly suggests that nicotine addiction is maintained through a motivational switch from positive reinforcement and the activation of the a-processes that moderate reward to negative reinforcement and the activation of b-processes that moderate stress response. The purpose of this paper was to highlight how abstinence-induced anxiety functions through the activation of the CRF system and is at least partially responsible for nicotine addiction. Furthermore, this paper presented research findings supporting the targeting of the CRF system in pharmacological interventions for smoking cessation and nicotine addiction.

The first section of this paper described and evaluated current pharmacological interventions used for smoking cessation. Those interventions include nicotine replacement therapies through the use of nicotine gum, patches or bupropion and varenicline. Despite some modest success rates, health concerns seem to oppose the use of bupropion and varenicline, and evidence suggests that the efficacy of nicotine gum and patches is not as high as marketing strategies suggest. Nicotine vaccinations have also been considered for the treatment of nicotine addiction, but a combination of concerns over their efficacy and cost-effectiveness has stalled research in this area. This leaves a gap in the treatment of nicotine addiction.

The second section of this paper highlighted how b-processes work through within and between system adaptations. Within system adaptations refer to neural changes in brain areas implicated in the rewarding properties of drugs and include attenuation of activity of the mesolimbic dopaminergic system and reduction in the number of D2 receptors in the striatum. On the other hand, between system adaptations refer to changes in brain regions other than the ones implicated in reward and include the extended amygdala, the HPA axis and CRF system.

The third and final section of this paper delved deeper into the workings of the CRF system. It was introduced that there are two types of $\mathrm{CRF}$ receptors; the $\mathrm{CRF}_{1}$ receptor that is thought to have an anxiogenic effect and the $\mathrm{CRF}_{2}$ receptor which is thought to have an anxiolytic effect. This CRF system is activated during acquisition, maintenance and withdrawal and is implicated in particular with increased anxiety during abstinence, which is suggested to lead to increased nicotine seeking behavior and likelihood of nicotine reinstatement. The results suggest that blocking $\mathrm{CRF}_{1}$ receptors could be an effective way of treating nicotine addiction. Preliminary animal studies support this idea, and administration of $\mathrm{CRF}_{1}$ receptor antagonist is associated with reduced anxietylike behaviors, hyperalgesia and nicotine seeking behaviors during abstinence. Despite the lack of human clinical studies testing the efficacy of $\mathrm{CRF}_{1}$ inhibitors for smoking cessation, research has shown that they have a modest therapeutic efficacy when used for anxiolytic purposes.

The first limitation is that, to the best of our knowledge, there is no clinical or pre-clinical study investigating the efficacy of $\mathrm{CRF}_{1}$ antagonists in humans attempting to quit smoking. Furthermore, no $\mathrm{CRF}_{1}$ antagonists have successfully completed the Phase III trial for the treatment of any psychiatric illness in gen- eral either (Zorilla et al., 2013). Additionally, another limitation is the extensive use of animal studies since nicotine addiction in humans is quite different than the operational definition of nicotine dependence in most animal studies. Humans self-administer nicotine through inhalation whereas, in most animal studies, nicotine is administered through the use of mini-pumps or injections in a non-contingent manner. Another limitation of the animal model of nicotine dependence is the frequent use of the short access model, in which animals are exposed to nicotine for only one hour a day (Cohen et al., 2013). However, humans smoke for most of the day and in a consistent pattern, indicating that they are at least partially aware when the bioavailability of nicotine in their bloodstream is reduced and the onset of nicotine withdrawal is eminent. In other words, humans may smoke every few hours in order to replenish nicotine levels in their bloodstream and attenuate the oncoming withdrawal syndrome.

That being said, this line of research is promising and opens several future directions. Firstly, researchers should attempt to emulate human smoking habits in animal studies. One study has already attempted to do this by allowing subjects to self-administer nicotine and providing these animals access to it for a longer period of time (Cohen et al., 2015). However, in this study, the animals used had undergone an invasive brain surgery. As such, it is likely that their smoking behaviors were affected by the stress associated with the surgery. Therefore, a future study should measure self-administration of nicotine in animals that have access to the stimulant for longer periods of time and have not been exposed to significant stressors. Furthermore, researchers using animal models of nicotine dependence could investigate how the self-administration acquisition time is correlated with anxiety symptoms during enforced abstinence and nicotine consumption at the end of abstinence. In other words, researchers could see if the animals that "learn" to self-administer nicotine faster are more anxious during the enforced abstinence period and consume more nicotine once they are given access to it again. Additionally, a number of sex-related dimorphisms in the experience and expression of stress have been observed (Weiten and McCann, 2006). Specifically, women are thought to be more likely to engage in internalizing behaviors, like rumination, whereas men are suggested to be more likely to engage in externalizing behaviors (Weiten and McCann, 2006). This is reflected by the fact that men have higher rates of antisocial behaviors and substance abuse, including smoking (Weiten and McCann, 2006). Therefore, a study investigating sex dimorphisms in nicotine withdrawal is needed. Finally, the administration of MPZP and other CRF blockers is limited by the fact that invasive surgery is required for it. However, it is unlikely that human participants would agree to undergo an invasive surgery to quit smoking, when other less dangerous alternatives exist. Thus, the production of a pharmacological agent using MPZP in the form of a pill or inhalant may be a necessary alternative.

Stress-induced smoking reinstatement is well-established and one of the most common reasons why cessation attempts fail. Therefore, targeting anxiety and stress directly with pharmacother- 
apy seems like a logical solution. Since $\mathrm{CRF}_{1}$ receptor antagonists reduce the dysphoric and stressful symptoms of both withdrawal and abstinence-induced anxiety, they could potentially be an effective pharmaceutical intervention for smoking cessation. This paper has presented research findings that support this idea and concludes that, despite the need for more research, $\mathrm{CRF}_{1}$ appear to be a promising alternative to current therapeutic interventions for nicotine dependence.

\section{REFERENCES}

American Psychiatric Association. (2013). Diagnostic and statistical manual of mental disorders (5th edition)

Bailey JE, Papadopoulos A, Diaper A, Phillips S, Schmidt ME, van der Ark P, ... \& Nutt DJ. (2011). Preliminary evidence of anxiolytic effects of the CRF1 receptor antagonist R317573 in the 7.5\% CO2 proof-of-concept experimental model of human anxiety. Journal of Psychopharmacology, 25(9), 1199-1206.

Baliunas D, Patra J, Rehm J, Popova S, Kaiserman M, \& Taylor B. (2007). Smoking-attributable mortality and expected years of life lost in Canada 2002: Conclusions for prevention and policy. Chronic Diseases and Injuries in Canada, 27(4), 154.

Benowitz NL. (2008). Neurobiology of nicotine addiction: implications for smoking cessation treatment. The American Journal of Medicine, 121(4), S3-S10.

Boorse GC \& Denver RJ. (2006). Widespread tissue distribution and diverse functions of corticotropin-releasing factor and related peptides. General and Comparative Endocrinology, 146(1), 9-18.

Brielmaier J, McDonald CG, \& Smith RF. (2012). Effects of acute stress on acquisition of nicotine conditioned place preference in adolescent rats: a role for corticotropin-releasing factor 1 receptors. Psychopharmacology, 219(1), 73 82.

Breslau N, Kilbey MM, \& Andreski P. (1992). Nicotine withdrawal symptoms and psychiatric disorders: findings from an epidemiologic study of young adults. The American Journal of Psychiatry, 149(4), 464.

Brondolo E, Monge A, Agosta J, Tobin JN, Cassells A, Stanton C, \& Schwartz, J. (2015). Perceived ethnic discrimination and cigarette smoking: examining the moderating effects of race/ethnicity and gender in a sample of Black and Latino urban adults. Journal of Behavioral Medicine, 38(4), 689-700.

Bruijnzeel AW. (2012). Tobacco addiction and the dysregulation of brain stress systems. Neuroscience \& Biobehavioral Reviews, 36(5), 1418-1441.

Bruijnzeel AW, Ford J, Rogers JA, Scheick S, Ji Y, Bishnoi M, \& Alexander JC. (2012). Blockade of CRF1 receptors in the central nucleus of the amygdala attenuates the dysphoria associated with nicotine withdrawal in rats. Pharmacology Biochemistry and Behavior, 101(1), 62-68.

Caggiula AR, Donny EC, Palmatier MI, Liu X, Chaudhri N, \& Sved AF. (2008). The role of nicotine in smoking: a dual-reinforcement model. In The Motivational Impact of Nicotine and its Role in Tobacco Use (pp. 91-109). Springer, New York, NY.

Centers for Disease Control and Prevention (CDC). (2011). Quitting smoking among adults--United States, 2001-2010. MMWR. Morbidity and Mortality Weekly Report, 60(44), 1513.

Chaiton M, Diemert L, Cohen JE, Bondy SJ, Selby P, Philipneri A, \& Schwartz R. (2016). Estimating the number of quit attempts it takes to quit smoking successfully in a longitudinal cohort of smokers. BMJ open, 6(6), e011045.

Christen A, Beiswanger B, Mau M, Walker C, Hatsukami D, Allen S, \& Cheney R. (1991). Transdermal nicotine for smoking cessation: six-month results from two multicenter controlled clinical trials. JAMA, 266(22), 3133-3138.

Cohen A, Treweek J, Edwards S, Leão RM, Schulteis G, Koob GF, \& George O. (2015). Extended access to nicotine leads to a CRF1 receptor dependent increase in anxiety-like behavior and hyperalgesia in rats. Addiction Biology, 20(1), 56-68.

Committee to Evaluate Drugs (2012). Varenicline - Ministry of Health and LongTerm Care Detailed Discussion: The CED reviewed varenicline in September 2007, May 2008, and February 2010. Key information considered by the CED throughout. Retrieved from: www.health.gov.on.ca/en/pro/programs/ drugs/ced/pdf/champix.pdf
Cornish KE, Harris AC, LeSage MG, Keyler DE, Burroughs D, Earley C, \& Pentel PR. (2011). Combined active and passive immunization against nicotine: minimizing monoclonal antibody requirements using a target antibody concentration strategy. International Immunopharmacology, 11(11), 1809-1815.

Dietz P. (2016). Physiology of Nicotine. In Tobacco Cessation and Substance Abuse Treatment in Women's Healthcare (pp. 25-32). Springer, Cham.

Etter JF \& Stapleton JA. (2006). Nicotine replacement therapy for long-term smoking cessation: a meta-analysis. Tobacco Control, 15(4), 280-285.

Fagerström KO, Schneider NG, \& Lunell E. (1993). Effectiveness of nicotine patch and nicotine gum as individual versus combined treatments for tobacco withdrawal symptoms. Psychopharmacology, 111(3), 271-277.

Feldman RS, Meyer JS, Quenzer LF, \& Cooper JR. (1997). Principles of Neuropsychopharmacology (No. 615+616 FEL). Sunderland: Sinauer Associates.

Garvey AJ, Bliss RE, Hitchcock JL, Heinold JW, \& Rosner B. (1992). Predictors of smoking relapse among self-quitters: a report from the Normative Aging Study. Addictive Behaviors, 17(4), 367-377.

George O, Ghozland S, Azar MR, Cottone P, Zorrilla EP, Parsons LH, \& Koob GF. (2007). CRF-CRF1 system activation mediates withdrawal-induced increases in nicotine self-administration in nicotine-dependent rats. Proceedings of the National Academy of Sciences, 104(43), 17198-17203.

Giri VP, Kanodia S, Giri OP, \& Sumit K. (2017). Anti-nicotine vaccine: current status. International Journal of Basic \& Clinical Pharmacology, 4(6), 13091313.

Gonzales D, Rennard SI, Nides M, Oncken C, Azoulay S, Billing CB, \& Varenicline Phase 3 Study Group. (2006). Varenicline, an $\alpha 4 \beta 2$ nicotinic acetylcholine receptor partial agonist, vs sustained-release bupropion and placebo for smoking cessation: a randomized controlled trial. JAMA, 296(1), 47-55.

Grieder TE, Herman MA, Contet C, Tan LA, Vargas-Perez H, Cohen A, \& Clarke L. (2014). CRF neurons in the ventral tegmental area control the aversive effects of nicotine withdrawal and promote escalation of nicotine intake. Nature Neuroscience, 17(12), 1751

Hatsukami DK, Rennard S, Jorenby D, Fiore M, Koopmeiners J, Vos A, \& Pentel PR. (2005). Safety and immunogenicity of a nicotine conjugate vaccine in current smokers. Clinical Pharmacology \& Therapeutics, 78(5), 456-467.

Health Canada. (2017). Canadian Tobacco Alcohol and Drugs (CTADS): 2015 Summary.

Henry B, Vale W, \& Markou A. (2006). The effect of lateral septum corticotropinreleasing factor receptor 2 activation on anxiety is modulated by stress. Journal of Neuroscience, 26(36), 9142-9152.

Hubbard R, Lewis S, West J, Smith C, Godfrey C, Smeeth L, \& Britton J. (2005). Bupropion and the risk of sudden death: a self-controlled case-series analysis using The Health Improvement Network. Thorax, 60(10), 848-850.

Hughes JR, Solomon LJ, Naud S, Fingar JR, Helzer JE, \& Callas PW. (2014). Natural history of attempts to stop smoking. Nicotine \& Tobacco Research, 16(9), 1190-1198.

Hughes JR, Stead LF, \& Lancaster T. (2007). Antidepressants for smoking cessation. Cochrane Database of Systematic Reviews, 1(1).

Johnson PM \& Kenny PJ. (2010). Dopamine D2 receptors in addiction-like reward dysfunction and compulsive eating in obese rats. Nature Neuroscience, 13(5), 635

Kamdi SP, Nakhate KT, Dandekar MP, Kokare DM, \& Subhedar NK. (2009). Participation of corticotropin-releasing factor type 2 receptors in the acute, chronic and withdrawal actions of nicotine associated with feeding behavior in rats. Appetite, 53(3), 354-362.

Koob GF. (2008). A role for brain stress systems in addiction. Neuron, 59(1), 11-34. Koob GF \& Volkow ND. (2010). Neurocircuitry addiction. Neuropsychopharmacology, 35(1), 217.

Kutlu MG, Holliday E, \& Gould TJ. (2016). High-affinity $\alpha 4 \beta 2$ nicotinic receptors mediate the impairing effects of acute nicotine on contextual fear extinction. Neurobiology of Learning and Memory, 128, 17-22.

Künzel HE, Zobel AW, Nickel T, Ackl N, Uhr M, Sonntag A, \& Holsboer F. (2003). Treatment of depression with the CRH-1-receptor antagonist R121919: endocrine changes and side effects. Journal of Psychiatric Research, 37(6), 525-533.

Lukoyanov NV, Madeira MD, \& Paula-Barbosa MM. (1999). Behavioral and neuroanatomical consequences of chronic ethanol intake and withdrawal. Physi- 
ology \& Behavior, 66(2), 337-346.

Macenski C. (2012). The Physiology of the HPA and Extended Amygdala in Mechanisms of Drug Use and Abuse.

Mason BJ, Crean R, Goodell V, Light JM, Quello S, Shadan F, Buffkins K, Kyle M, Adusumalli M, \& Rao S. (2012). A proof-of-concept randomized controlled study of gabapentin: effects on cannabis use, withdrawal and executive function deficits in cannabis-dependent adults. Neuropsychopharmacology, 37(7), 1689.

Mano-Otagiri A, Iwasaki-Sekino A, Ohata H, Arai K, \& Shibasaki T. (2009). Nicotine suppresses energy storage through activation of sympathetic outflow to brown adipose tissue via corticotropin-releasing factor type 1 receptor. $\mathrm{Neu}$ roscience Letters, 455(1), 26-29.

Murrough JW, Yaqubi S, Sayed S, \& Charney DS. (2015). Emerging drugs for the treatment of anxiety. Expert Opinion on Emerging Drugs, 20(3), 393-406.

Nichols HB \& Harlow BL. (2004). Childhood abuse and risk of smoking onset. Journal of Epidemiology \& Community Health, 58(5), 402-406.

Nides M, Oncken C, Gonzales D, Rennard S, Watsky EJ, Anziano R, \& Reeves KR. (2006). Smoking cessation with varenicline, a selective $\alpha 4 \beta 2$ nicotinic receptor partial agonist: results from a 7-week, randomized, placebo-and bupropion-controlled trial with 1-year follow-up. Archives of Internal Medicine, 166(15), 1561-1568.

Olive MF, Koenig HN, Nannini MA, \& Hodge CW. (2002). Elevated extracellular CRF levels in the bed nucleus of the stria terminalis during ethanol withdrawal and reduction by subsequent ethanol intake. Pharmacology Biochemistry and Behavior, 72(1-2), 213-220.

O’Loughlin JL, Dugas EN, O'Loughlin EK, Karp I, \& Sylvestre MP. (2014). Incidence and determinants of cigarette smoking initiation in young adults. Journal of Adolescent Health, 54(1), 26-32.

Ontario Tobacco Research Unit. 2013 Annual Strategy Monitoring Report. Toron to: Ontario Tobacco Research Unit

Park PE, Vendruscolo LF, Schlosburg JE, Edwards S, Schulteis G, \& Koob GF. (2013). Corticotropin-releasing factor (CRF) and $\alpha 2$ adrenergic receptors mediate heroin withdrawal-potentiated startle in rats. International Journal of Neuropsychopharmacology, 16(8), 1867-1875.

Pedersen W \& Soest TV. (2017). How is low parental socioeconomic status associated with future smoking and nicotine dependence in offspring? A population-based longitudinal 13-year follow-up. Scandinavian Journal of Public Health, 45(1), 16-24.

Pentel PR \& LeSage MG. (2014). New directions in nicotine vaccine design and use. In Advances in Pharmacology (Vol. 69, pp. 553-580). Academic Press.

Pentel PR, Dufek MB, Roiko SA, Lesage MG, \& Keyler DE. (2006). Differential effects of passive immunization with nicotine-specific antibodies on the acute and chronic distribution of nicotine to brain in rats. Journal of Pharmacology and Experimental Therapeutics, 317(2), 660-666.

Pravetoni M, Keyler DE, Raleigh MD, Harris AC, Lesage MG, Mattson CK, ... \& Pentel PR. (2011). Vaccination against nicotine alters the distribution of nicotine delivered via cigarette smoke inhalation to rats. Biochemical Pharmacology, 81(9), 1164-1170.

Ramiro LS, Madrid BJ, \& Brown DW. (2010). Adverse childhood experiences (ACE) and health-risk behaviors among adults in a developing country setting. Child Abuse \& Neglect, 34(11), 842-855.

Richter RM \& Weiss F. (1999). In vivo crf release in rat amygdala is increased during cocaine withdrawal in self-administering rats. Synapse, 32(4), 254-261.

Rodaros D, Caruana DA, Amir S, \& Stewart J. (2007). Corticotropin-releasing factor projections from limbic forebrain and paraventricular nucleus of the hypothalamus to the region of the ventral tegmental area. Neuroscience, 150(1), 8-13.

Schmidt ME, Andrews RD, van der Ark P, Brown T, Mannaert E, Steckler T, \& Van Laere K. (2010). Dose-dependent effects of the CRF 1 receptor antagonist R317573 on regional brain activity in healthy male subjects. Psychopharmacology, 208(1), 109.

Schnoll RA, Goelz PM, Veluz-Wilkins A, Blazekovic S, Powers L, Leone FT, \& Hitsman B. (2015). Long-term nicotine replacement therapy: a randomized clinical trial. JAMA Internal Medicine, 175(4), 504-511.

Shiffman S, Johnston JA, Khayrallah M, Elash CA, Gwaltney CJ, Paty JA, \& DeVeaugh-Geiss J. (2000). The effect of bupropion on nicotine craving and withdrawal. Psychopharmacology, 148(1), 33-40.

Silva SM \& Madeira MD. (2012). Effects of chronic alcohol consumption and withdrawal on the response of the male and female hypothalamic-pituitaryadrenal axis to acute immune stress. Brain Research, 1444, 27-37.

Singh S, Loke YK, Spangler JG, \& Furberg CD. (2011). Risk of serious adverse cardiovascular events associated with varenicline: a systematic review and meta-analysis. Canadian Medical Association Journal, cmaj-110218.

Skinner MD \& Aubin HJ. (2010). Craving's place in addiction theory: contributions of the major models. Neuroscience \& Biobehavioral Reviews, 34(4), 606-623.

Slawecki CJ, Thorsell AK, El Khoury A, Mathé AA, \& Ehlers CL. (2005). Increased CRF-like and NPY-like immunoreactivity in adult rats exposed to nicotine during adolescence: relation to anxiety-like and depressive-like behavior. Neuropeptides, 39(4), 369-377.

Smith SM \& Vale WW. (2006). The role of the hypothalamic-pituitary-adrenal axis in neuroendocrine responses to stress. Dialogues in Clinical Neuroscience, 8(4), 383.

Subramaniyan M \& Dani JA. (2015). Dopaminergic and cholinergic learning mechanisms in nicotine addiction. Annals of the New York Academy of Sciences, 1349(1), 46-63.

Thorsell A, Repunte-Canonigo V, O'Dell LE, Chen SA, King AR, Lekic D, ... \& Paolo Sanna P. (2007). Viral vector-induced amygdala NPY overexpression reverses increased alcohol intake caused by repeated deprivations in Wistar rats. Brain, 130(5), 1330-1337.

Tsatsanis C, Dermitzaki E, Venihaki M, Chatzaki E, Minas V, Gravanis A, \& Margioris AN. (2007). The corticotropin-releasing factor (CRF) family of peptides as local modulators of adrenal function. Cellular and Molecular Life Sciences, 64(13), 1638-1655.

US Department of Health and Human Services. (2014). The health consequences of smoking - 50 years of progress: a report of the Surgeon General. Atlanta, GA: US Department of Health and Human Services, Centers for Disease Control and Prevention, National Center for Chronic Disease Prevention and Health Promotion, Office on Smoking and Health, 17.

US Food and Drug Administration Early communication about an ongoing safety review of varenicline (marketed as Chantix) Nov 20, 2010. Available at: http://www.fda.gov/Drugs/DrugSafety/PostmarketDrugSafetyInformationforPatientsandProviders/DrugSafetyInformationforHeathcareProfessionals/ ucm070765.htm. Accessed March 24, 2018

US Food and Drug Administration Public Health Advisory: FDA requires new boxed warnings for the smoking cessation drugs Chantix and Zyban. Jul 1, 2009. Available at:http://www.fda.gov/Drugs/DrugSafety/PublicHealthAdvisories/ucm169988.htm. Accessed March 24, 2018

Vale W, Spiess J, Rivier C, \& Rivier J. (1981). Characterization of a 41-residue ovine hypothalamic peptide that stimulates secretion of corticotropin and $\beta$-endorphin. Science, 1394-1397.

Volkow ND, Fowler JS, Wang GJ, Baler R, \& Telang F. (2009). Imaging dopamine's role in drug abuse and addiction. Neuropharmacology, 56, 3-8.

Weiten W \& McCann D. (2016). "Psychology: themes and variations", Fourth Canadian edition. Toronto: Nelson Education.

West R, Baker CL, Cappelleri JC, \& Bushmakin AG. (2008). Effect of varenicline and bupropion SR on craving, nicotine withdrawal symptoms, and rewarding effects of smoking during a quit attempt. Psychopharmacology, 197(3), 371-377.

Wilkes S. (2008). The use of bupropion SR in cigarette smoking cessation. International Journal of Chronic Obstructive Pulmonary Disease, 3(1), 45.

Wise RA \& Koob GF. (2014). The development and maintenance of drug addiction. Neuropsychopharmacology, 39(2), 254.

Zalewska-Kaszubska, J. (2015). Is immunotherapy an opportunity for effective treatment of drug addiction? Vaccine, 33(48), 6545-6551.

Zhao Y, Valdez GR, Fekete EM, Rivier JE, Vale WW, Rice KC, \& Zorrilla EP. (2007). Subtype-selective corticotropin-releasing factor receptor agonists exert contrasting, but not opposite, effects on anxiety-related behavior in rats Journal of Pharmacology and Experimental Therapeutics, 323(3), 846-854.

Zorrilla EP, Valdez GR, \& Weiss F. (2001). Changes in levels of regional CRF-likeimmunoreactivity and plasma corticosterone during protracted drug withdrawal in dependent rats. Psychopharmacology, 158(4), 374-381. 